\title{
A Comparative Study on Consensus Measures in Group Decision
}

\author{
Making \\ María José del Moral \\ Department of Statistics and Operational Research, University of Granada, Spain \\ Email: delmoral@ugr.es \\ Francisco Chiclana* \\ Centre for Computational Intelligence, De Montfort University, Leicester, UK \\ Email: chiclana@dmu.ac.uk \\ Juan Miguel Tapia \\ Department of Quantitative Methods in Economic and Business, University of Granada,Spain \\ Email: jmtaga@ugr.es \\ Enrique Herrera-Viedma ${ }^{\dagger}$ \\ Department of Computer Science, University of Granada, Spain \\ Email: viedma@decsai.ugr.es
}

June 19, 2017

\begin{abstract}
In group decision making problems it is desirable to obtain a high level of consensus among experts before reaching a solution. It is customary to construct consensus measures by using similarity functions to quantify the closeness of experts preferences. In such process the use of a metric that describes the distance between experts preferences allows the definition of similarity and dissimilarity -distancefunctions. Different distance functions have been proposed in order to implement consensus measures. This paper examines how the use of different aggregation operators affects the level of consensus achieved by experts through different distance functions, once the number of experts has been established in

* Corresponding author

†Corresponding author
\end{abstract}


the decision making problem. The experimental study conducted states that the speed of the consensus process is significantly affected by the use of diverse aggregation operators and distance functions. Several decision support rules which can be useful in controling the convergence speed of the consensus process are also derived.

Keywords: Group Decision Making; Fuzzy Preferences; Similarity; Consensus; Decision Support Rules.

\section{Introduction}

In those decision situations in which several individuals are involved, Group Decision Making (GDM) problems, each member of the group, referred to as expert, recognizes the existence of a common problem and tries to come to a collective decision. For reaching such decision, experts express their preferences through a set of evaluations over a set of possible alternatives. It is expected that the final decision may be reached thanks to a wide enough agreement among experts. 1 These agreed decisions have led to the well-known concept of consensus, which has emerged as a topic of increasing interest. 24

It is usual to define consensus as the total and unanimous agreement of all the experts in relation to the feasible alternatives $\sqrt[5]{6}$ This definition may become a drawback since it only allows to differentiate between two states, existence or absence of consensus. Another significance of the concept of consensus refers to the judgement arrived at by 'most of' those concerned, which has led to the introduction of a new concept of consensus degree referred to as 'soft' consensus degree $4 / 7$ 7 8

Using such soft consensus measure, the consensus process can be described as a dynamic process with iterative group discussion rounds, coordinated by a moderator, which helps the members of the group to make their opinions closer $2 \sqrt{2}[10$ Some consensus processes have been proposed using an information procedure, such as a feedback mechanism which provides experts with visual representations of their consensus positions, $11[12$ or a recommendation mechanism to generate personalised advices when facing disparate opinions of multiple experts $!^{13}$ Dong et al ${ }^{14}$ proposed a dynamic consensus model by means of a self-management mechanism that generates experts' weights dynamically and integrated it into the consensus reaching process. The same authors $\frac{15}{15}$ in the context where decision makers have different interests and they use individual sets of attributes to evaluate the individual alternatives, developed a consensus process that generates adjustment suggestions for individual sets of attributes, individual sets of alternatives and individual preferences, thus helping decision makers reach consensus.

In order to evaluate the consensus it is necessary the computation and aggregation of the distance rep-

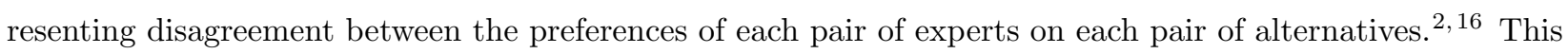
aggregation operation allows to obtain a fuzzy majority representation. The aggregator most widely used in 
GDM problems is the weighted average ordered (OWA) operator, $9[17$ and different extension of this aggregator has been proposed 18 20 A fundamental issue here is to determine the weights associated to the OWA operator. $\mathrm{Xu} \stackrel{21}{21}$ reviewed the main existing methods for determining the weights and developed a practical method for obtaining the OWA weights, a normal distribution based method. In order to model dynamism in the consensus processes some authors have proposed the inclusion of a temporal variable (t) in the model and several aggregation operators have been introduced in order to aggregate information $\sqrt[22] 23]{23}$ With this aim $\mathrm{Xu}{ }^{24}$ introduced the dynamic weighted averaging (DWA) operator and adapted this operator to treat different types of information such as real numbers, interval numbers, or linguistic labels, defining the concepts of argument variable and dynamic weighted geometric aggregation (DWGA) operator ${ }^{25}$ Following the same line $\mathrm{Xu}$ and Yager ${ }^{26}$ proposed a dynamic intuitionistic fuzzy weighted averaging (DIFWA) operator and an uncertain dynamic intuitionistic fuzzy weighted averaging (UDIFWA) operator.

The level of agreement among experts is represented by soft consensus measures and their definition is based on the concept of similarity between their preferences. An important question to be addressed at this point is that the convergence of the consensus process towards an acceptable solution could be affected by the specific distance function and the aggregation operator used to measure disagreement $\sqrt[27]{32}$

Applying the non-parametric Wilcoxon test, Chiclana et al $!^{33}$ found significant differences between the behaviour of five of the most commonly used distance functions in modelling soft consensus measures, 34 Manhattan, Euclidean, Cosine, Dice, and Jaccard. This behaviour were further analysed using a convergent criterion. Finally a set of rules were identified for their application to control the speed of convergence towards consensus. In this framework, the aim of this paper is to analyze how the use of different aggregation operators (Maximum, Minimum and Average) affects the level of consensus achieved by experts through the different distance functions, once the number of experts has been established in the GDM problem.

This paper is structured as follows. Section 2 introduces concepts essential to the understanding of the rest of the paper: the GDM problem (Subsection 2.1), the selection process (Subsection 2.2) and the consensus process (Subsection 2.3). Section 3 describes the design of the experiment and the results obtained. Finally, Section 4 concludes the paper.

\section{Preliminaries}

This section describes the common framework to work out consensus processes in GDM problems. 


\subsection{The GDM Problem}

In a GDM context, a group of experts, $E=\left\{e_{1}, \ldots, e_{m}\right\}(m \geq 2)$, through their experience and awareness, express their preferences on a set of viable alternatives, $X=\left\{x_{1}, \ldots, x_{n}\right\}(n \geq 2)$, in order to come to a collective decision (Figure 1). Millet ${ }^{35}$ studied several preference elicitation methods and a comparison among some of them shown that pairwise procedures are preferable to non-pairwise ones.

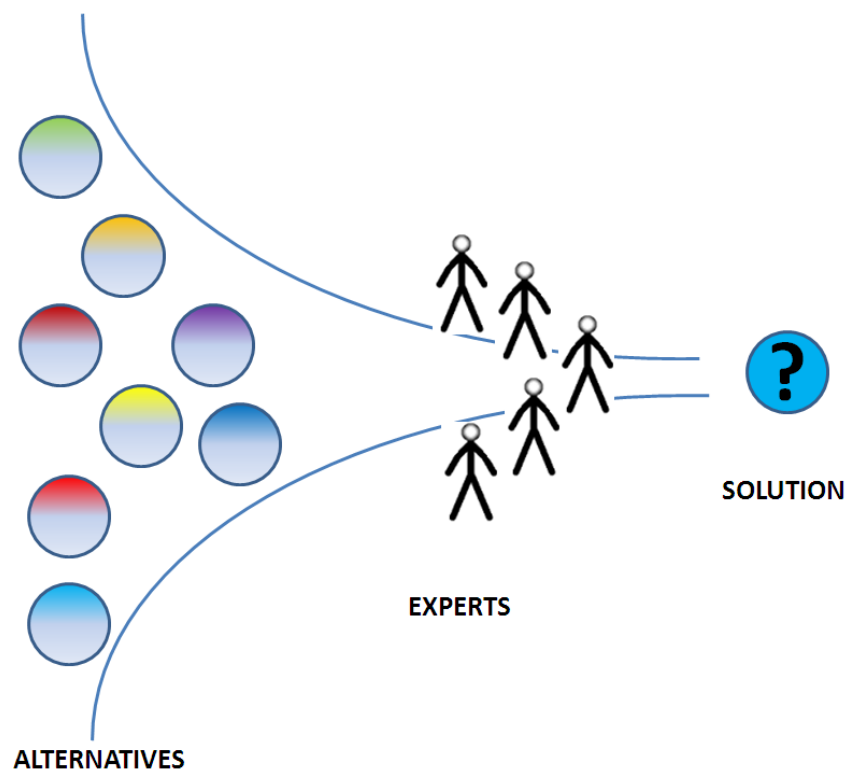

Figure 1: A GDM Problem

Fuzzy sets theory has revealed as a very useful tool in describing experts's preferences on $X$ in GDM problems $\sqrt[33[36]{36} \text { In a fuzzy environment, }$

Definition 1. Given a finite set of alternatives $X$, a fuzzy preference relation $P$ on $X$ is defined by a function $\mu_{P}: X \times X \longrightarrow[0,1], \mu\left(x_{i}, x_{j}\right)=p_{i j}$, that verifies

$$
p_{i j}+p_{j i}=1 \forall i, j \in\{1, \ldots, n\} .
$$

Every value $p_{i j}$ represents the preference degree of $x_{i}$ over $x_{j}: p_{i j}=1$ indicates that $x_{i}$ is absolutely preferred to $\left.x_{j}, p_{i j} \in\right] 0.5,1\left[\right.$ indicates that $x_{i}$ is preferred to $x_{j}$ and $p_{i j}=0.5$ indicates indifference between $x_{i}$ and $x_{j}$. $P$ can be denoted by a matrix $P=\left(p_{i j}\right)$ when $X$ has a small cardinality (Figure 2).

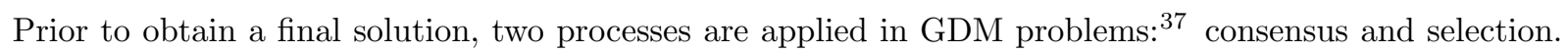
The first one is intended to obtain the maximum degree of consensus or agreement among the experts. The second one provides the final solution according to the preferences provided by the experts. 


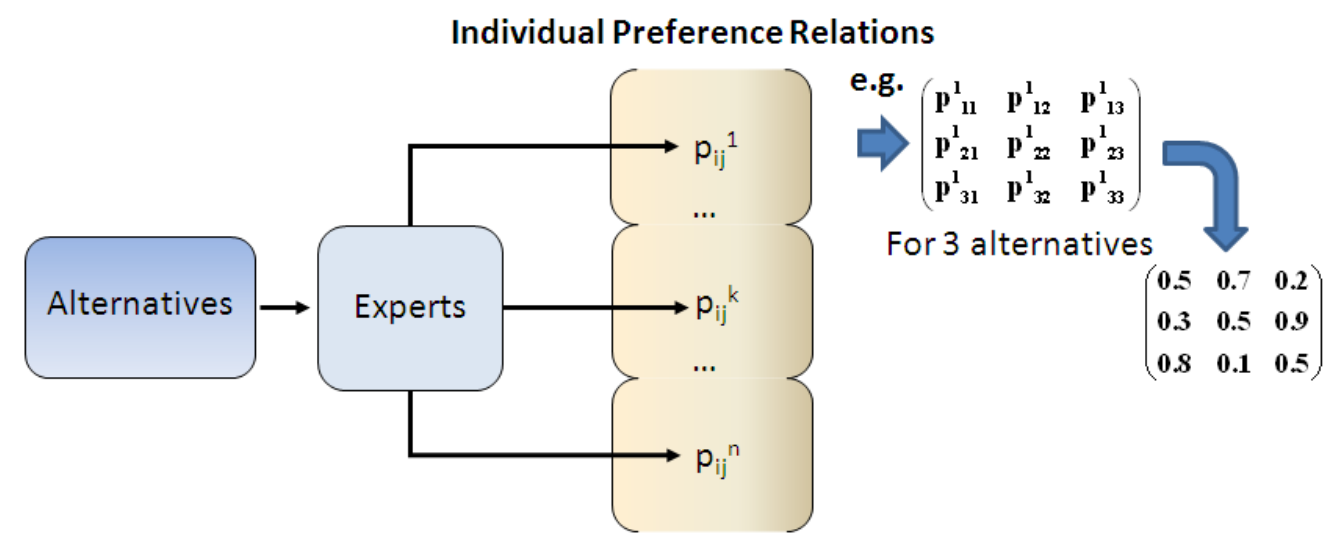

Figure 2: Fuzzy Preference Relation

\subsection{Selection Process}

Usually the selection process consists of two different phases, aggregation and exploitation $38 \mid 39$

Aggregation phase. In theis phase a collective preference relation, $P^{c}=\left(p_{i j}^{c}\right)$, is defined by means of the aggregation of all individual fuzzy preference relations $\left\{P^{1}, P^{2}, \ldots, P^{m}\right\}$ (Figure 3). The collective preference relation indicates the global preference between every pair of alternatives agreed by the majority of experts.

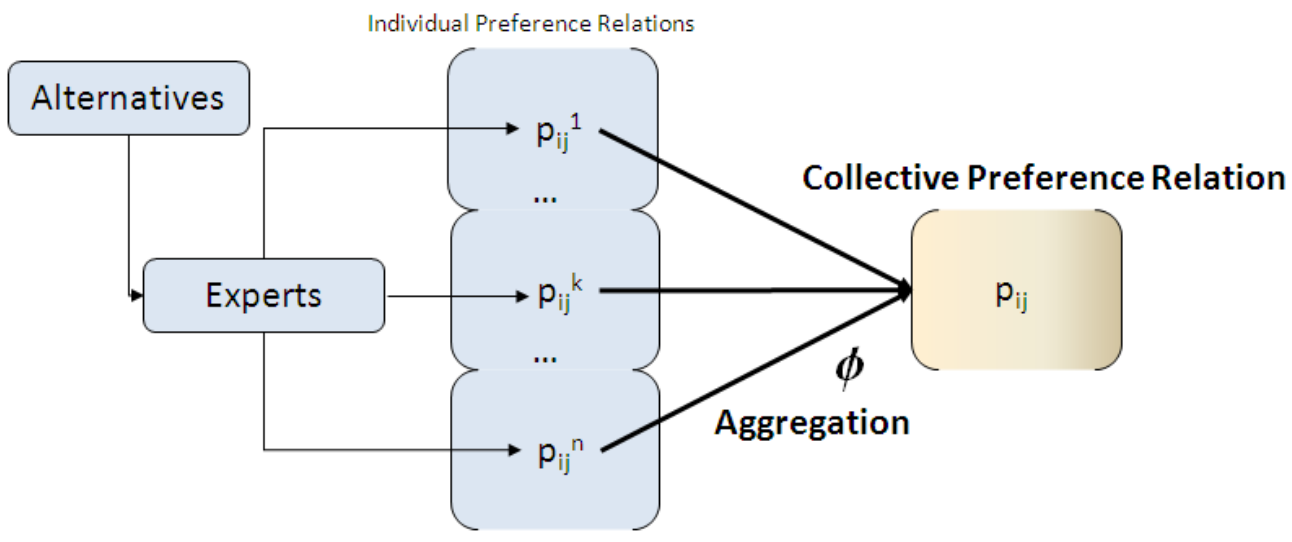

Figure 3: Aggregation Phase

In the aforementioned process plays a fundamental role the concept of fuzzy majority, a soft concept of majority which can be managed by calculating linguistically quantified propositions ${ }^{40}$ and also by making use of ordered weighted aggregation (OWA) operators $17 \mid 19$

Specifically, the aggregation operation through a quantifier guided OWA operator, $\phi_{Q}$, is performed as below:

$$
p_{i j}^{c}=\phi_{Q}\left(p_{i j}^{1}, \ldots, p_{i j}^{m}\right)=\sum_{k=1}^{m} w_{k} \cdot p_{i j}^{\sigma(k)}
$$


where $\sigma$ denotes a permutation function according to which

$$
p_{i j}^{\sigma(k)} \geq p_{i j}^{\sigma(k+1)}, \forall k=1, \ldots, \mathrm{m}-1
$$

and $Q$ is a fuzzy linguistic quantifier ${ }^{[0]}$ representing the concept of fuzzy majority and that is used to calculate the weighting vector of $\phi_{Q}: W=\left(w_{1}, \ldots, w_{n}\right), w_{k} \in[0,1], \sum_{k=1}^{n} w_{k}=1$, according to the expression! 17

$$
w_{k}=Q(k / n)-Q((k-1) / n), \forall k \in\{1, \ldots, n\}
$$

$$
Q(r)= \begin{cases}0 & \text { if } 0 \leq r<a \\ \frac{r-a}{b-a} & \text { if } a \leq r \leq b \\ 1 & \text { if } b<r \leq 1\end{cases}
$$

The concept of fuzzy majority has been treated in the literature and admits different representations $\sqrt[4]{19}[22$

Exploitation phase. This phase transforms the global information about the alternatives into a global ranking of them and a set of alternatives is obtained as the solution of the problem.

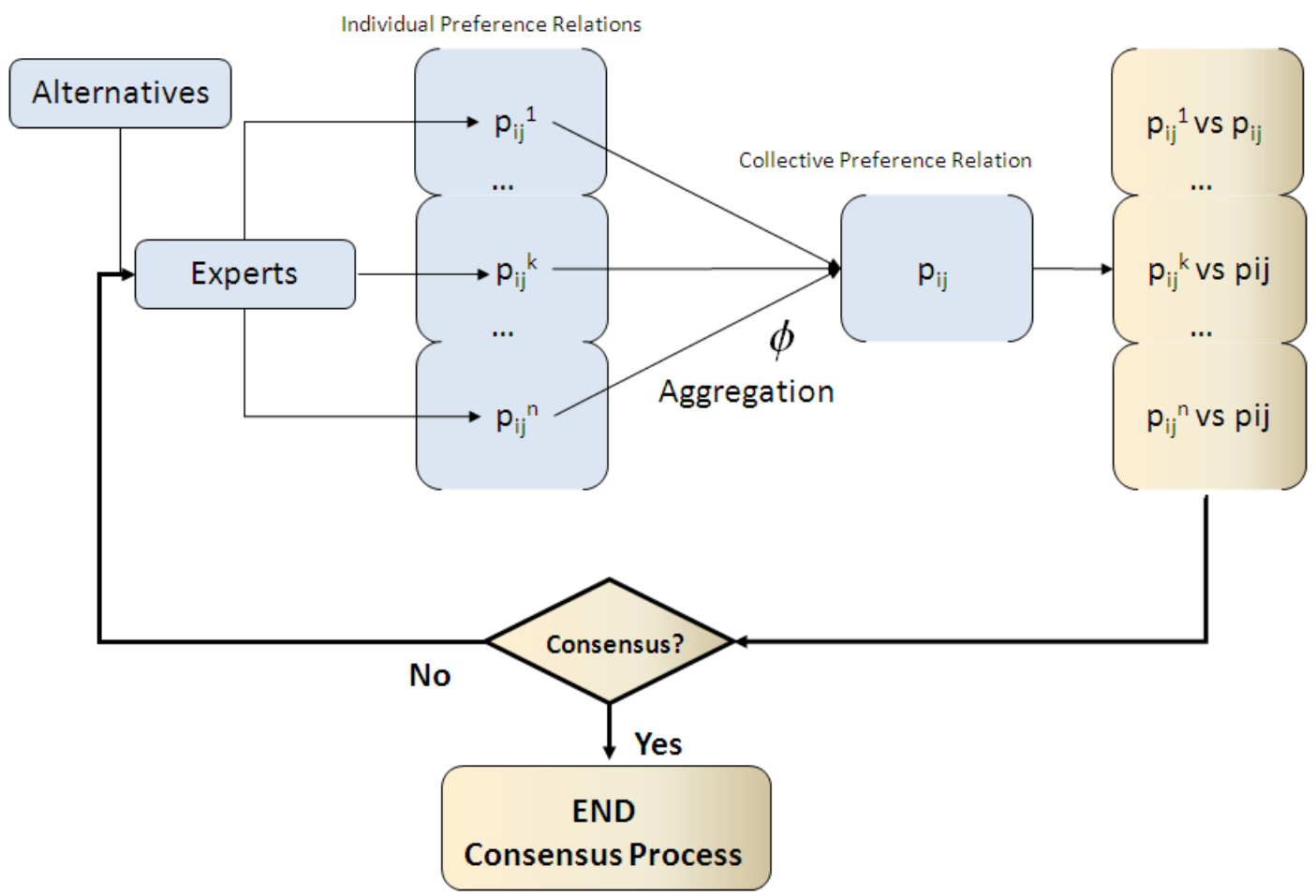

Figure 4: Consensus Model 
It is expected that the final decision in a GDM problem may be reached thanks to a high level of consensus among experts' in relation to their preferences (Figure 4).

\subsection{Consensus Model}

To measure the level of agreement among experts it is necessary to measure the distance or, equivalently, the similarity between their preference values. Let us now formalize these concepts. ${ }^{34}$

Definition 2. Let $A$ be a set. A function $d: A \times A \longrightarrow \mathbb{R}$ is called a distance (or dissimilarity) on $A$ if, for all $x, y \in A$, there holds

1. $d(x, y) \geq 0$ (non-negativity)

2. $d(x, y)=d(y, x)$ (symmetry)

3. $d(x, x)=0$ (reflexivity)

Some of the most commonly used distance functions are the following $33 \mid 34$

Definition 3. Given two vectors of real numbers $\mathbf{a}=\left(a_{1}, \ldots, a_{n}\right)$ and $\mathbf{b}=\left(b_{1}, \ldots, b_{n}\right)$, the Manhattan distance function is given by:

$$
d_{1}(\mathbf{a}, \mathbf{b})=\sum_{i=1}^{n}\left|a_{i}-b_{i}\right|
$$

the Euclidean distance function is written:

$$
d_{2}(\mathbf{a}, \mathbf{b})=\sqrt{\sum_{i=1}^{n}\left|a_{i}-b_{i}\right|^{2}}
$$

the Cosine distance function is defined by:

$$
d_{3}(\mathbf{a}, \mathbf{b})=\frac{\sum_{i=1}^{n} a_{i} \cdot b_{i}}{\sqrt{\sum_{i=1}^{n} a_{i}^{2}} \cdot \sqrt{\sum_{i=1}^{n} b_{i}^{2}}}
$$

the Dice distance function is expressed as:

$$
d_{4}(\mathbf{a}, \mathbf{b})=\frac{2 \cdot \sum_{i=1}^{n} a_{i} \cdot b_{i}}{\sum_{i=1}^{n} a_{i}^{2}+\sum_{i=1}^{n} b_{i}^{2}}
$$


and the Jaccard distance function:

$$
d_{5}(\mathbf{a}, \mathbf{b})=\frac{\sum_{i=1}^{n} a_{i} \cdot b_{i}}{\sum_{i=1}^{n} a_{i}^{2}+\sum_{i=1}^{n} b_{i}^{2}-\sum_{i=1}^{n} a_{i} \cdot b_{i}}
$$

Definition 4. Let $A$ be a set. A function $s: A \times A \longrightarrow \mathbb{R}$ is called a similarity on $A$ if $s$ is non-negative, symmetric, and if $s(x, y) \leq s(x, x)$ holds for all $x, y \in A$, with equality if and only if $x=y$.

The main transformations between a distance $d$ and a similarity $s$ bounded by 1 are $33 \mid 34$

$$
d=1-s ; d=\frac{1-s}{s} ; d=\sqrt{1-s} ; d=\sqrt{2\left(1-s^{2}\right)} ; d=\arccos s ; d=-\ln s
$$

The similarity function allows to measure consensus degrees and proximity measures $\frac{10}{10}$ The consensus degrees are derived by fusing the similarity of the preference values of all the experts on each pair of alternatives by the expression (11). The proximity measures are obtained by quantifying the similarity between the preferences of each expert in the group and the collective preferences, previously calculated by joining all the individual experts' preferences.

One of the principal problems is to find a way to make individual positions converge and support the experts in the agreement to obtain a particular solution. Because of this, a consensus level for that solution is previously stablished.

The degrees of consensus are derived from the following steps:

1. A similarity matrix, $S M^{r}=\left(s m_{i j}^{r}\right)$, records the proximity between the preference values provided by each expert, $r$, and the corresponding preference values of the rest of the experts in the group, with

$$
s m_{i j}^{r}=s\left(\mathbf{p}_{\mathbf{i j}}^{\mathbf{r}}, \mathbf{p}_{\mathbf{i j}}\right)
$$

where $\mathbf{p}_{\mathbf{i j}}^{\mathbf{r}}=\left(p_{i j}^{r}, \ldots, p_{i j}^{r}\right), \mathbf{p}_{\mathbf{i j}}=\left(p_{i j}^{1}, \ldots, p_{i j}^{r-1}, p_{i j}^{r+1}, \ldots, p_{i j}^{m}\right)$ and $s:[0,1]^{m-1} \times[0,1]^{m-1} \rightarrow[0,1]$ is a similarity function. The closer $s m_{i j}^{r}$ to 1 , the more similar $\mathbf{p}_{\mathbf{i j}}^{\mathbf{r}}$ and $\mathbf{p}_{\mathbf{i j}}$ are, while the closer $s m_{i j}^{r}$ to 0 , the more distant $\mathbf{p}_{\mathbf{i j}}^{\mathbf{r}}$ and $\mathbf{p}_{\mathbf{i j}}$ are.

2. A consensus matrix, $C M=\left(c m_{i j}\right)$, is obtained by aggregating, using an OWA operator $(\phi)$, all the similarity matrices obtained via Equation (11):

$$
\forall i, j \in\{1, \ldots, n\}: c m_{i j}=\phi\left(s m_{i j}^{1}, \ldots, s m_{i j}^{m}\right)
$$




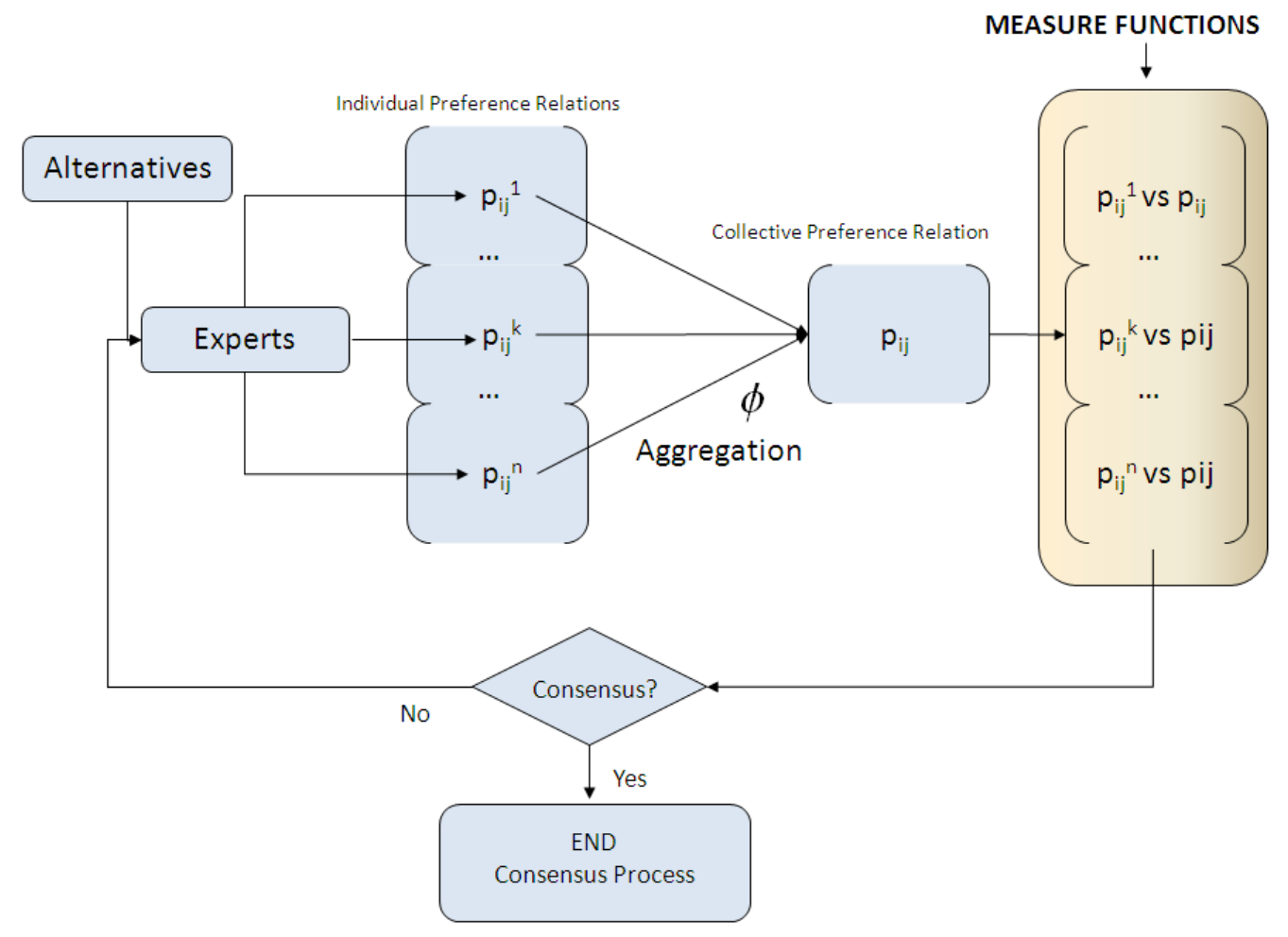

Figure 5: Applying Measure Functions

3. Consensus degrees are defined in each one of the three different levels of a fuzzy preference relation! $\frac{38}{6}$

Level 1. Consensus on the pairs of alternatives, $c p_{i j}$. It measures the agreement among all experts on the pair of alternatives $\left(x_{i}, x_{j}\right)$ :

$$
\forall i, j=1, \ldots, n \wedge i \neq j: c p_{i j}=c m_{i j}
$$

Level 2. Consensus on alternatives, $\mathrm{ca}_{i}$. It measures the agreement among all experts on the alternative $x_{i}$, and it is obtained by aggregating the consensus degrees of all the pairs of alternatives involving it:

$$
c a_{i}=\phi\left(c p_{i j}, c p_{j i} ; j=1, \ldots, n \wedge j \neq i\right)
$$

Level 3. Consensus on the relation, $c r$. It measures the global agreement among all experts, and it is obtained by aggregating all the consensus degrees at the level of pairs of alternatives:

$$
c r=\phi\left(c a_{i} ; i=1, \ldots, n\right)
$$




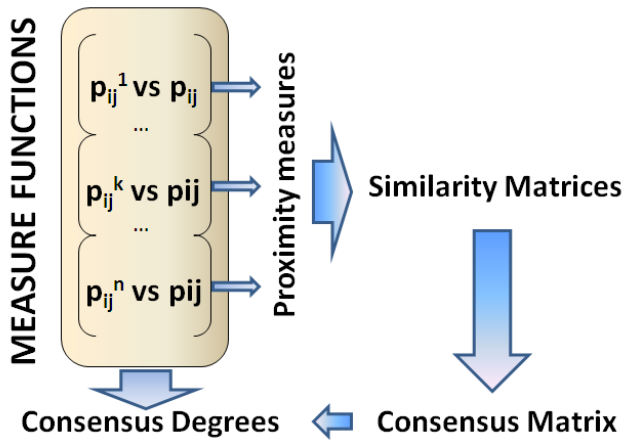

Figure 6: Measure Computation

\section{Comparative Study: Experimental Design and Result}

In Chiclana et al $\stackrel{33}{5}$ a study on the effect of the application of the distance functions given in Definition 3 was carried out. Using the non-parametric Wilcoxon's matched-pairs signed-ranks test, it was shown not only that different distance functions could produce significantly different results, but also its application has a significant effect on the speed in the consensus process. So we start by assuming the hypothesis corroborated in! $! 33$

"The application of the Manhattan, Euclidean, Cosine, Dice and Jaccard distance functions in GDM problems produce significant differences in the measurement of consensus"

In this study we intend to analyze, once the number of experts has been established, how the use of different OWA operators affects the level of consensus achieved through the different distance functions. Consensus degrees are deduced at the Level 3 of a fuzzy preference relation, that is, at the level of the relation. We consider three OWA operators:

- Maximum, with weighting vector $W=\left(w_{1}, \ldots, w_{n}\right), w_{1}=1$ and $w_{j}=0$ for $j \neq 1$,

- Minimum, with weighting vector $W=\left(w_{1}, \ldots, w_{n}\right), w_{n}=1$ and $w_{j}=0$ for $j \neq n$, and

- Average, with weighting vector $W=\left(w_{1}, \ldots, w_{n}\right), w_{j}=1 / n$ for all $j, 1 \leq j \leq n$.

To that end, twelve randomized GDM problems have been generated, one for each of the possible combinations of experts $(m=4,6,8,10)$ and alternatives $(n=4,6,8)$. Each one of these random GDM problems have been executed three times, each time using one of the three different OWA operators given above in order to compute the consensus degrees at the level of the relation.

Table 3 shows the level of consensus (in percentage) reached by the number of experts considered for each GDM problem. The higher this value in this table, the greater the global level of consensus achieved by the 
experts in the corresponding GDM problem. For each number of experts, comparison of column entries could be used to find out which OWA operator and distance function returns the largest values and, therefore, could lead to a faster convergence in the consensus process.

Figure 7 displays the evolution of the degree of consensus in percentage according to the number of experts for the three OWA operators and so do the Figures 8, 9 and 10 for each of the OWA operators separately.

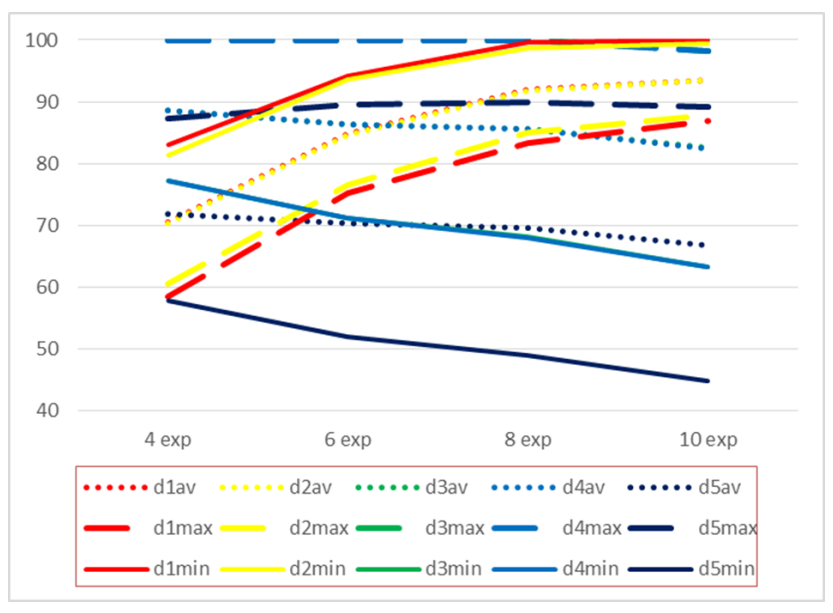

Figure 7: Evolution of the degree of consensus in percentage according to the number of experts for the three OWA operators.

Some points can be emphasized from the results shown in Table 3.

1. Four experts: Cosine $\left(d_{3}\right)$ and Dice $\left(d_{4}\right)$ distance functions with Maximum OWA obtain the highest values. The differences with Manhattan $\left(d_{1}\right)$ and Euclidean $\left(d_{2}\right)$ distance functions are between $10 \%$ and $30 \%$, reaching in some cases $40 \%$ with Maximum OWA. Jaccard distance function $\left(d_{5}\right)$ is located between both groups, except for the Minimum OWA.

2. Six experts: The highest values are obtained in a similar way. However, the differences with Manhattan $\left(d_{1}\right)$ and Euclidean $\left(d_{2}\right)$ distance functions are reduced, being between $5 \%$ and $15 \%$. Jaccard distance function $\left(d_{5}\right)$ is the distance with lower values not only with the Minimum OWA, but also with the average OWA.

3. Eight experts: The behavior is similar to that of the cases with four and six experts, although further reducing the differences. Jaccard distance function $\left(d_{5}\right)$ has a similar behavior to the case of six experts, but the differences being greater.

4. Ten experts: Manhattan $\left(d_{1}\right)$ and Euclidean $\left(d_{2}\right)$ distance functions outperform the rest of the distance functions with the minimum OWA. The differences are between $2 \%$ and $35 \%$. Jaccard distance function $\left(d_{5}\right)$ behaves similarly to the case of eight experts. 


\begin{tabular}{llllll}
\hline \multicolumn{7}{c}{ "Average" OWA } \\
\hline Experts $\backslash d_{i}$ & $d_{1}$ & $d_{2}$ & $d_{3}$ & $d_{4}$ & $d_{5}$ \\
\hline 4 & 70,53 & 70,33 & 88,66 & 88,58 & 71,82 \\
6 & 84,85 & 84,74 & 86,39 & 86,29 & 70,26 \\
8 & 91,96 & 91,87 & 85,66 & 85,57 & 69,60 \\
10 & 93,59 & 93,52 & 82,49 & 82,39 & 66,71 \\
\hline \multicolumn{7}{c}{} \\
\hline \multicolumn{7}{c}{ "Maximum" OWA } \\
\hline Experts $\backslash d_{i}$ & $d_{1}$ & $d_{2}$ & $d_{3}$ & $d_{4}$ & $d_{5}$ \\
\hline 4 & 58,45 & 60,50 & 100,0 & 99,91 & 87,26 \\
6 & 75,27 & 76,55 & 100,0 & 99,95 & 89,50 \\
8 & 83,32 & 85,05 & 100,0 & 99,91 & 89,90 \\
10 & 86,87 & 87,93 & 98,20 & 98,15 & 89,17 \\
\hline \multicolumn{7}{c}{} \\
\hline Experts $\backslash d_{i}$ & $d_{1}$ & $d_{2}$ & $d_{3}$ & $d_{4}$ & $d_{5}$ \\
\hline 4 & 83,03 & 81,28 & 77,24 & 77,20 & 57,79 \\
6 & 94,20 & 93,65 & 71,20 & 71,11 & 52,05 \\
8 & 99,56 & 98,64 & 68,13 & 68,06 & 48,97 \\
10 & 100,0 & 99,47 & 63,32 & 63,27 & 44,75 \\
\hline \multicolumn{7}{c}{}
\end{tabular}

Table 1: Consensus degree in percentages for all GDM problems at the level of the relation

\subsection{Convergence Rules for the Consensus Process}

As a result of the previous analysis, some rules can be drawn in order to speed up or slow down the convergence of the consensus and that could become an important tool to support decision making in GDM problems.

- Four experts: To achieve a fast consensus process Cosine $\left(d_{3}\right)$ and Dice $\left(d_{4}\right)$ distance functions could be used with the Maximum OWA. While if a slow consensus process is preferred, it would be advisable to choose Jaccard distance function $\left(d_{5}\right)$ with the Minimum OWA or, also, Manhattan $\left(d_{1}\right)$, Euclidean $\left(d_{2}\right)$ or Jaccard $\left(d_{5}\right)$ distance functions with the Average OWA.

- Six experts: Cosine $\left(d_{3}\right)$ and Dice $\left(d_{4}\right)$ distance functions could be used with the Maximum OWA to reach a fast consensus or, also, Manhattan $\left(d_{1}\right)$ and Euclidean $\left(d_{2}\right)$ distance functions with the Minimum OWA or Jaccard $\left(d_{5}\right)$ distance function with the Maximun OWA. When a slow consensus process is searched, Jaccard distance function $\left(d_{5}\right)$ would be chosen with the Minimum OWA or, also, Cosine $\left(d_{3}\right)$ and Dice $\left(d_{4}\right)$ distance functions with the Minimum OWA or, in addition, Jaccard $\left(d_{5}\right)$ distance function with the Average OWA.

- Eight experts: Cosine $\left(d_{3}\right)$ and Dice $\left(d_{4}\right)$ distance functions could be used with the Maximum OWA to get a fast consensus process or, also, Manhattan $\left(d_{1}\right)$ and Euclidean $\left(d_{2}\right)$ distance functions with the 


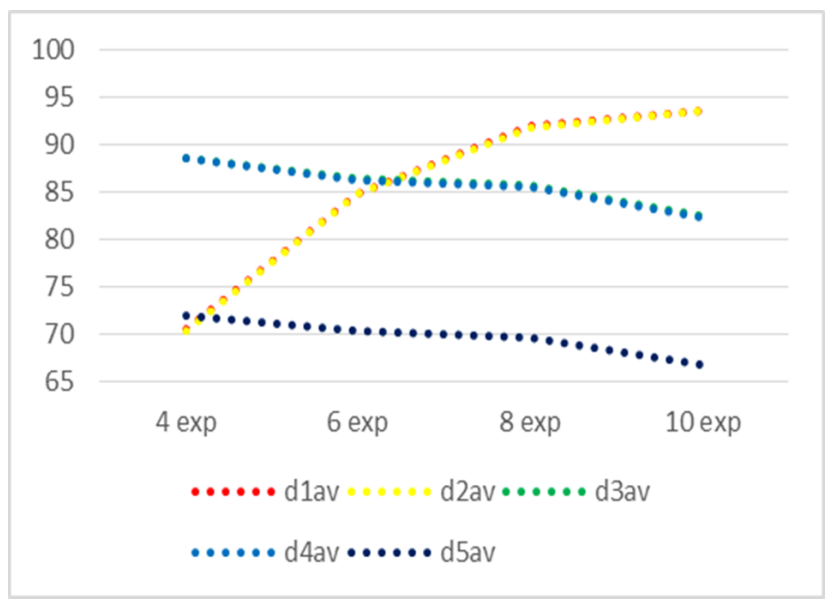

Figure 8: Evolution of the degree of consensus in percentage according to the number of experts for the "Average" OWA operator.

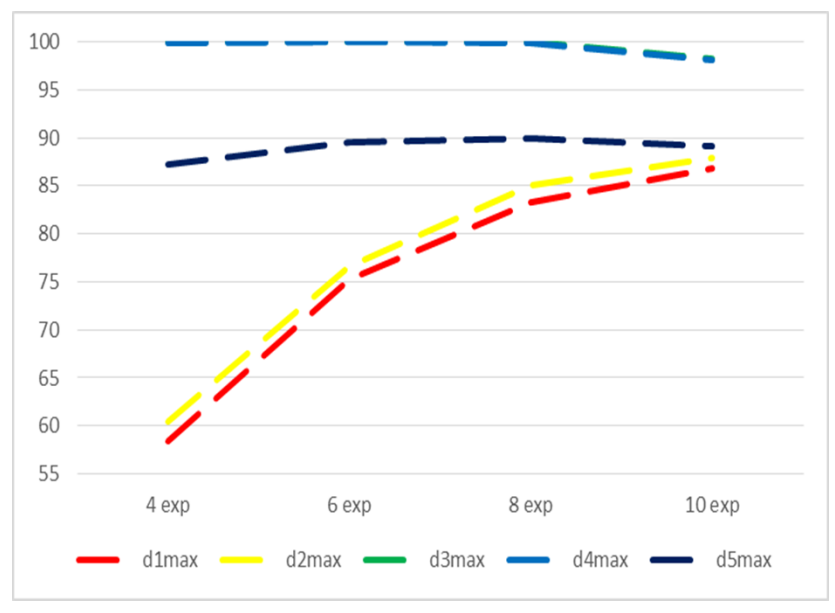

Figure 9: Evolution of the degree of consensus in percentage according to the number of experts for the "Maximum" OWA operator.

Minimum OWA or, even, Manhattan $\left(d_{1}\right.$ and Euclidean $\left(d_{2}\right)$ distance functions with the Average OWA. But if a slow consensus process is desired, it would be advisable to choose Jaccard distance function $\left(d_{5}\right)$ with Minimum OWA or, also, Jaccard $\left(d_{5}\right)$ distance function with Average OWA.

- Ten experts: To reach a fast consensus, Manhattan $\left(d_{1}\right)$ and Euclidean $\left(d_{2}\right)$ distance functions could be used with the Minimum OWA, or Cosine $\left(d_{3}\right)$ and Dice $\left(d_{4}\right)$ distance functions with the Maximum OWA. Also the choice could be Manhattan $\left(d_{1}\right)$ and Euclidean $\left(d_{2}\right)$ distance functions with the Average OWA. However, when a slow consensus process is requested, it would be recommended to choose Jaccard $\left(d_{5}\right)$ distance function with the Minimum OWA or, also, Cosine $\left(d_{3}\right)$ and Dice $\left(d_{4}\right)$ distance functions with the Minimum OWA, or Jaccard $\left(d_{5}\right)$ distance functions with the Average OWA.

A summary of these rules is shown in Table 2 . 


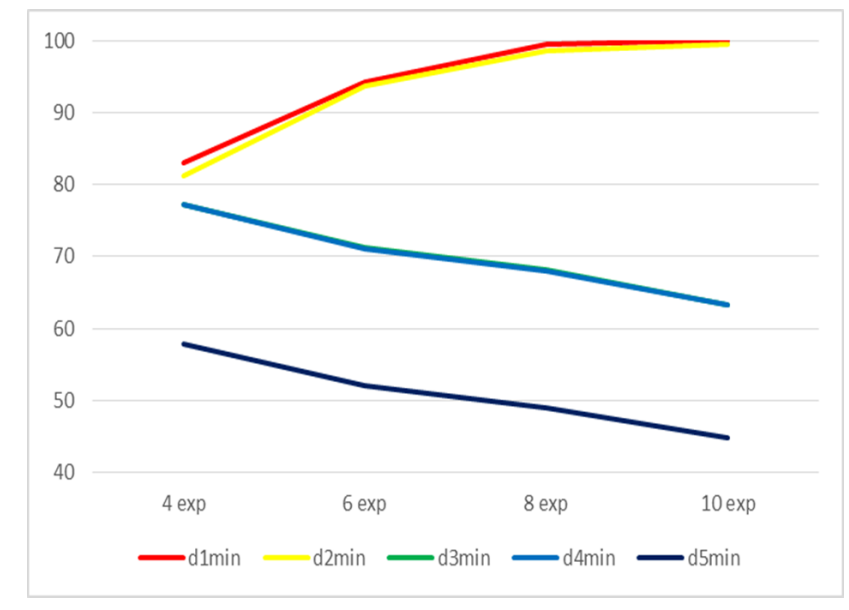

Figure 10: Evolution of the degree of consensus in percentage according to the number of experts for the "Minimum" OWA operator.

\begin{tabular}{cccccc}
\hline & \multicolumn{2}{c}{ Fast Consensus Process } & & \multicolumn{2}{c}{ Slow Consensus Process } \\
\cline { 2 - 3 } \cline { 5 - 6 } For & Highest & High & & Lowest & Low \\
\hline 4 exp. & $d_{3} / d_{4}$ (Maximum) & $d_{5}$ (Maximum) & & $d_{5}$ (Minimum) & $\begin{array}{c}d_{1} / d_{2} / d_{5} \\
\text { (Average) }\end{array}$ \\
\hline 6 exp. & $d_{3} / d_{4}$ (Maximum) & $d_{1} / d_{2}$ (Minimum) & & $d_{5}$ (Minimum) & $d_{3} / d_{4}$ (Minimum) \\
& & $d_{5}$ (Maximum) & & $d_{5}$ (Average) \\
\hline 8 exp. & $d_{3} / d_{4}$ (Maximum) & $d_{1} / d_{2}$ (Average) & & $d_{5}$ (Minimum) & $d_{5}$ (Average) \\
& $d_{1} / d_{2}$ (Minimum) & & & \\
\hline 10 exp. & $d_{3} / d_{4}$ (Maximum) & $d_{1} / d_{2}$ (Average) & & $d_{5}$ (Minimum) & $d_{3} / d_{4}$ (Minimum) \\
& $d_{1} / d_{2}$ (Minimum) & & & $d_{5}$ (Average) \\
\hline
\end{tabular}

Table 2: Summary of the Convergence Rules - Distance (OWA)

As an illustrative example, we perform a GDM problem considering the four possible values for the number of experts in our study, using the three aggregation operators with the five different distance functions. We record the number of rounds needed for the consensus process to reach the threshold consensus level accepted for the GDM problem. This is graphically represented in Figures 11, 12, 13 and 14 .

\section{Conclusions}

In this paper we have analyzed how the use of different OWA operators -Maximum, Minimum and Average OWA operators- affects the level of consensus achieved through five of the most commonly used distance functions, Manhattan, Euclidean, Cosine, Dice and Jaccard, once the number of experts of the GDM problem has been established. Consensus degrees are deduced at the level of the relation.

The results in our experimental study have shown that, according to the number of experts considered, the aggregation operators and distance functions produce significantly different results in most of the GDM 


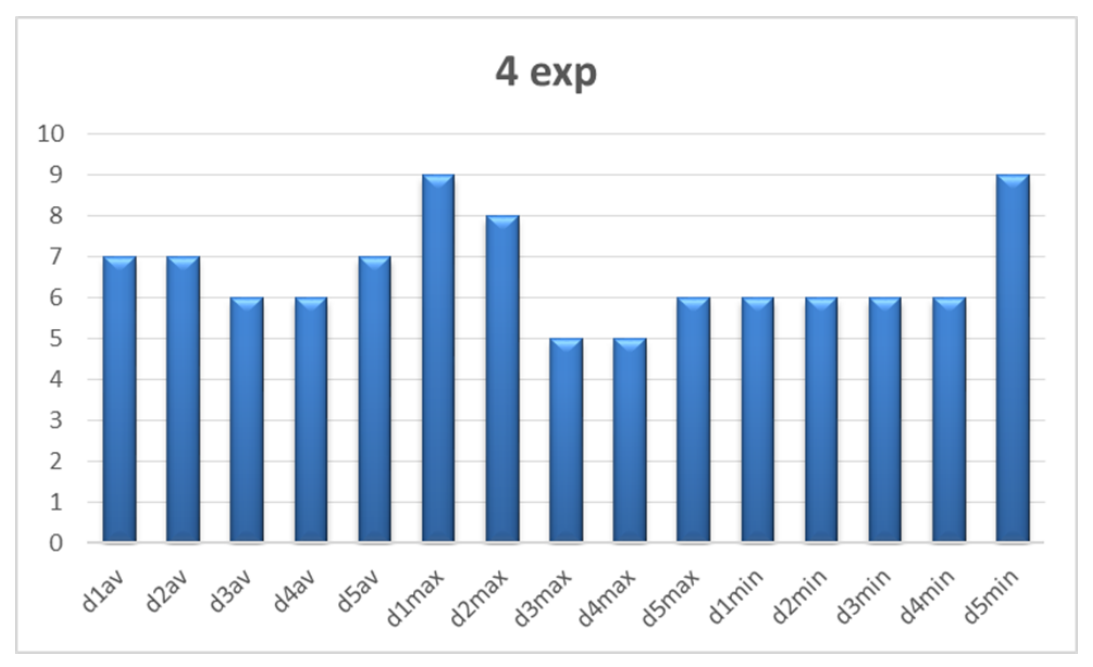

Figure 11: Number of rounds needed for each distance function to reach the consensus threshold in the GDM problem: 4 experts

problems carried out. The analysis of the outcomes allows to draw a set of rules that can be used to control the convergence speed of the consensus process and could become an important tool to support decision making in GDM problems.

\section{Acknowledgement}

This research work has been supported by the research projects grants (TIN2013-40658-P and TIN201675850-R) from the FEDER funds.

\section{References}

[1] Butler, C.T., Rothstein, A. On Conflict and Consensus: A Handbook on Formal Consensus Decision Making. Takoma Park, 2006.

[2] Cabrerizo, F.J., Moreno, J.M., Pérez, I.J., Herrera-Viedma, E. Analyzing consensus approaches in fuzzy group decision making: advantages and drawbacks. Soft Computing, 14(5):451-463, 2010.

[3] Dong, Y.C., Xu,J. Consensus building in group decision making: Searching the consensus path with minimum adjustments. Springer, 2016.

[4] Herrera-Viedma, E., Cabrerizo, F.J., Kacprzyk, J., Pedrycz, W. A Review of Soft Consensus Models in a Fuzzy Environment. Information Fusion, 17:4-13, 2014. 


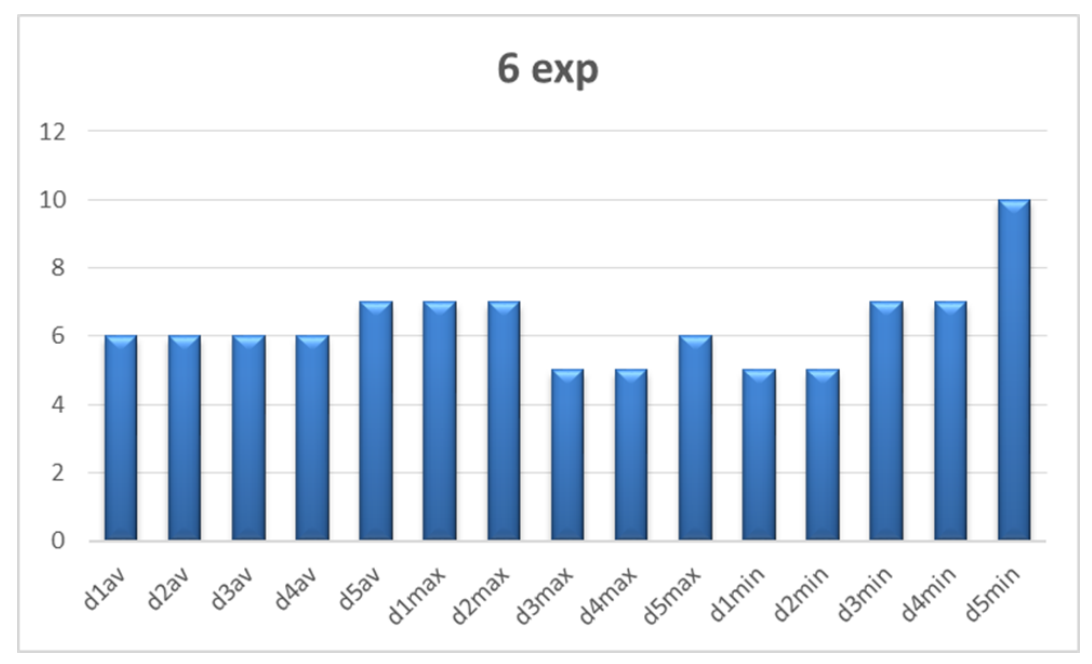

Figure 12: Number of rounds needed for each distance function to reach the consensus threshold in a GDM problem: 6 experts

[5] Bezdek, J.C., Spillman, B., Spillman, R. A fuzzy relation space for group decision theory. Fuzzy Sets and Systems, 1(4):255-268, 1978.

[6] Spillman, B., Spillman, R., Bezdek, J.C. A fuzzy analysis of consensus in small groups. Wang, P.P., Chang, S.K. (Eds.), Fuzzy Automata and Decision Processes, North-Holland, Amsterdam, 1980, pp. $331-356$.

[7] Cabrerizo, F. J., Chiclana, F., Al-Hmouz, R., Morfeq, A., Balamash, A. S., Herrera-Viedma, E. Fuzzy decision making and consensus: challenges. Journal of Intelligent \& Fuzzy Systems, 29(3):1109-1118, 2015.

[8] Kacprzyk, J., Fedrizzi, M. 'Soft' consensus measures for monitoring real consensus reaching processes under fuzzy preferences. Control and Cybernetics, 15:309-323, 1986.

[9] Fedrizzi, M., Kacprzyk, J., Nurmi, H. Consensus degrees under fuzzy majorities and fuzzy preferences using OWA (ordered weighted average) operators. Control and Cybernetics, 22:71-80, 1993.

[10] Herrera, F., Herrera-Viedma, E., Verdegay, J.L. A model of consensus in group decision making under linguistic assessments. Fuzzy Sets and Systems, 78(1):73-87, 1996.

[11] Wu, J., Chiclana, F. Visual information feedback mechanism and attitudinal prioritisation method for group decision making with triangular fuzzy complementary preference relations. Information Sciences, 279:716-734, 2014.

[12] Wu, J., Chiclana, F., Fujita, H., Herrera-Viedma, E. A visual interaction consensus model for social network group decision making with trust propagation. Knowledge-Based Systems, 122:39-50, 2017. 


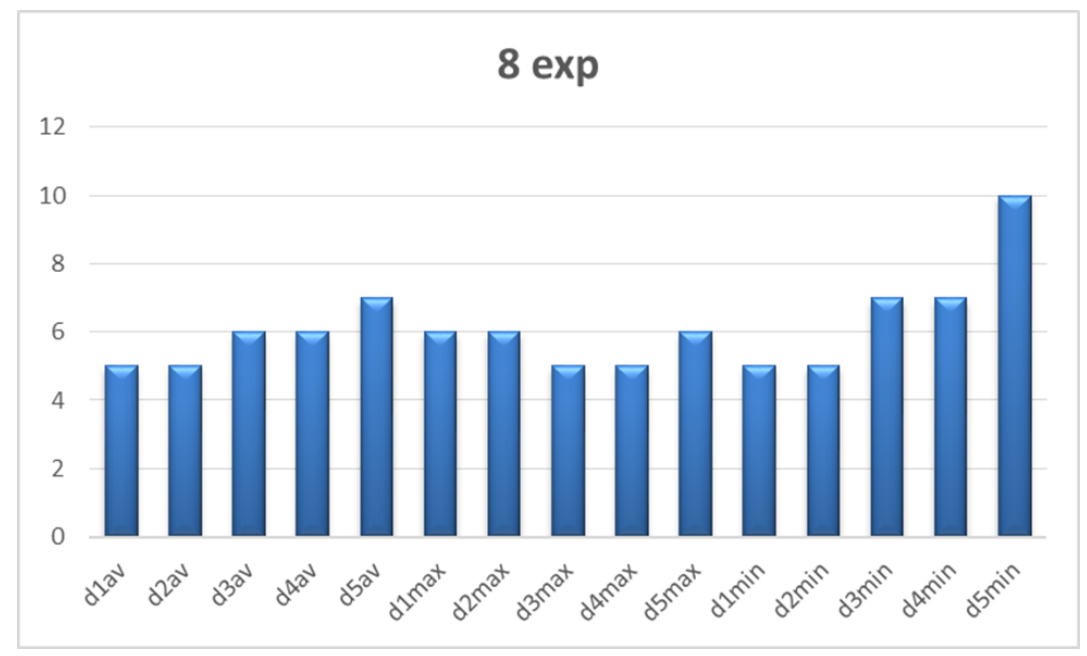

Figure 13: Number of rounds needed for each distance function to reach the consensus threshold in a GDM problem: 8 experts

[13] Liu, Y., Liang, C., Chiclana, F., Wu, J. A trust induced recommendation mechanism for reaching consensus in group decision making. Knowledge-Based Systems, 119:221-231, 2017.

[14] Dong, Y.C., Zhang, H., Herrera-Viedma, E. Integrating experts' weights generated dynamically into the consensus reaching process and its applications in managing non-cooperative behaviors. Decision Support Systems, 84(1):1-15, 2016.

[15] Dong, Y.C., Zhang, H., Herrera-Viedma, E. Consensus reaching model in the complex and dynamic MAGDM problem. Knowledge-Based Systems 106(1):206-219, 2016.

[16] Pérez, I.J., Cabrerizo, F.J., Alonso, S., Herrera-Viedma, E. A New Consensus Model for Group Decision Making Problems with Non Homogeneous Experts', IEEE Transactions on Systems, Man, and Cybernetics: Systems, 44(4):494-498, 2014.

[17] Yager, R.R. On ordered weighted averaging aggregation operators in multicriteria decision making. IEEE Transactions on Systems, Man and Cybernetics, 18:183-190, 1988.

[18] Merigó, J.M., Guillén, M., Sarabia, J.M. The Ordered Weighted Average in the Variance and the Covariance. International Journal of Intelligent Systems, 30(9):985-1005, 2015

[19] Yager, R.R. Quantifier guided aggregation using OWA operators. International Journal of Intelligent Systems, 11:49-73, 1996.

[20] Yager, R.R., Alajlan, N. Some issues on the OWA aggregation with importance weighted arguments. Knowledge-Based Systems, 100:89-96, 2016. 


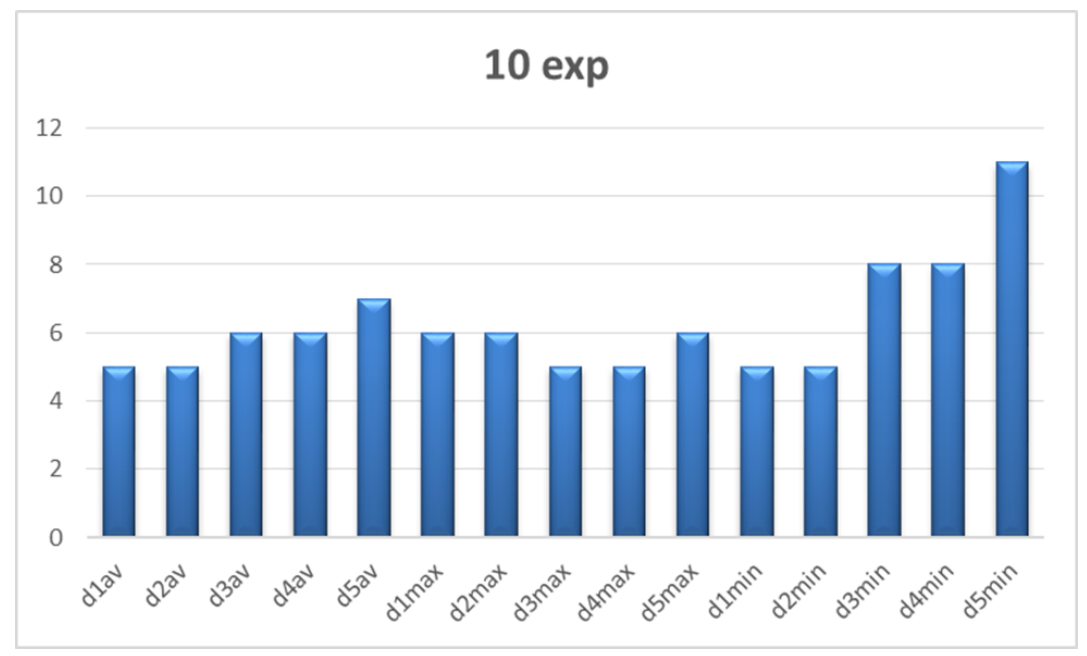

Figure 14: Number of rounds needed for each distance function to reach the consensus threshold in a GDM problem: 10 experts

[21] Xu, Z. An overview of methods for determining owa weights. International Journal of Intelligent Systems, 20(8):843-865, 2005.

[22] Yager, R.R., Kacprzyk, J. The ordered weighted averaging operators: theory and applications. Springer Science \& Business Media, 2012

[23] Kolter, J., Maloof, M. Dynamic weighted majority: An ensemble method for drifting concepts. Journal of Machine Learning Research, 8:2755-2790, 2007.

[24] Xu, Z. On multi-period multi-attribute decision making. Knowledge-Based Systems, 21(2):164-171, 2008.

[25] Xu, Z. A method based on the dynamic weighted geometric aggregation operator for dynamic hybrid multi-attribute group decision making. International Journal of Uncertainty, Fuzziness and KnowledgeBased Systems, 17(1):15-33, 2009.

[26] Xu, Z., Yager, R.R. Dynamic intuitionistic fuzzy multi-attribute decision making. International Journal of Approximate Reasoning, 48(1):246-262, 2008.

[27] Alonso, S., Pérez, I.J., Cabrerizo, F.J., Herrera-Viedma, E. A Linguistic Consensus Model for Web 2.0 Communities. Applied Soft Computing, 13(1):149-157, 2013.

[28] Cabrerizo, F.J., Morente-Molinera, J.A., Pérez, I.J., López-Gijón, J., Herrera-Viedma, E. A decision support system to develop a quality management in academic digital libraries. Information Sciences, 323:48-58, 2015. 
[29] Pérez, I.J., Cabrerizo, F.J., Herrera-Viedma, E. A Mobile Decision Support System for Dynamic Group Decision Making Problems. IEEE Transactions on Systems, Man and Cybernetics - Part A: Systems and Humans, 40(6):1244-1256, 2010.

[30] Tapia García, J.M., Del Moral Ávila, M.J., Martinez, M.A., Herrera-Viedma, E. A Consensus Model for Group Decision Making Problems with Linguistic Interval Fuzzy Preference Relations. Expert System With Applications, 39(11):10022-10030, 2012.

[31] Tapia García, J.M., Del Moral Ávila, M.J., Martinez, M.A., Herrera-Viedma, E. A Consensus Model for Group Decision Making Problems with Interval Fuzzy Preference Relations. International Journal of Information Technology \& Decision Making, 11(4):709-725, 2012.

[32] Wu, J., Chiclana, F., Herrera-Viedma, E. Trust Based Consensus Model for Social Network in an Incomplete Linguistic Information Context. Applied Soft Computing, 35:827-839, 2015.

[33] Chiclana, F., Tapia García, J.M., Del Moral Ávila, M.J., Herrera-Viedma, E. A statistical comparative study of different similarity measures of consensus in GDM. Information Sciences, 221:110-123, 2013.

[34] Deza, M.M., Deza, E. Encyclopedia of Distances. Springer, Berlin, Heidelberg, 2009.

[35] Millet, I. The effectiveness of alternative preference elicitation methods in the analytic hierarchy process. Journal of Multi-Criteria Decision Analysis, 6(1):41-51, 1997.

[36] Kacprzyk, J. Group decision making with a fuzzy linguistic majority. Fuzzy Sets and Systems, 18(2):105$118,1986$.

[37] Morente-Molinera, J.A., Wikström, R., Herrera-Viedma, E., Carlsson, C. A linguistic mobile decision support system based on fuzzy ontology to facilitate knowledge mobilization. Decision Support Systems, 81:66-75, 2016.

[38] Cabrerizo, F.J., Heradio, R., Pérez, I.J., Herrera-Viedma, E. A selection process based on additive consistency to deal with incomplete fuzzy linguistic information. Journal of Universal Computer Science, 16(1):62-81, 2010.

[39] Roubens, M. Fuzzy sets and decision analysis. Fuzzy Sets and Systems, 90(2):199-206, 1997.

[40] Zadeh, L.A. A computational approach to fuzzy quantifiers in natural languages. Computers $\&$ Mathematics with Applications, 9(1):149-184, 1983. 